\title{
Radiación gamma en rocas usadas como material de construcción: el Granito de Braga (No de Portugal)
}

\author{
Gamma Radiation In Rocks Used As Building \\ Materials: The Braga Granite (Nw Portugal)
}

LIMA, M. ${ }^{1}$, ALVES, C. ${ }^{1}$ and SANJURJO-SÁNCHEZ, J. ${ }^{2}$

(1) Lab2PT - Landscape, Heritage and Territory Laboratory/Centro de Investigação Geológica, Ordenamiento e Valorização de Recursos (Fundação para a Ciência e a Tecnologia, PEst-OE/CTE/ UI0697/2011 and PEst-OE/CTE/UI0697/2014), Campus de Gualtar, School of Sciences, University of Minho, Braga, Portugal

(2) University Institute of Geology "Isidro Parga Pondal”, University of A Coruña, A Coruña, Spain.

https://doi.org/10.17979/cadlaxe.2015.38.0.3684

\begin{abstract}
Granite can be a rock with significative radioactivity levels due to its content in radioisotopes of $\mathrm{K}$, $\mathrm{U}$ e Th. The Braga granite has been extensively used from the past in historical buildings of this city in North Portugal. A gamma radiation assessment has been made in quarry samples of the Braga granite with different weathering degree, in order to assess radiation risks in historical buildings, derived from their content in radionuclides. The specific activities of radioisotopes in ${ }^{238} \mathrm{U}$ and ${ }^{232} \mathrm{Th}$ decay chains and radioactive ${ }^{40} \mathrm{~K}$ were measured by gamma spectrometry on samples with different weathering degree. The results were used to calculate the gamma building index $(I ;$; EU technical guidance RP112) a other indices to estimate the radiological hazard of the rock as building material. Specific activities are above world averages and all the samples have $\mathrm{I}_{\gamma}>1$ (the threshold that implies specific studies for building materials). This result can be especially relevant for historical building where this granite is used as bulk material.
\end{abstract}


KEYWORDS: gamma radiation, radioisotopes, granite, gamma spectrometry, historical buildings

\section{Agradecimiento}

La "Fundação para a Ciência e a Tecnologia" de Portugal financia las Unidades de Investigación Lab2PT - Landscape, Heritage and Territory Laboratory y el Centro de Investigação Geológica, Ordenamiento e Valorização de Recursos (este último por los proyectos PEst-OE/CTE/UI0697/2011 and PEst-OE/CTE/UI0697/2014). 


\section{INTRODUCCIÓN}

La radiación natural es considerada de lejos como la principal responsable de la dosis de radiación ionizante externa que recibe la población durante su vida (UNSCEAR, 2000). Por ello, es extremadamente importante conocer las dosis de radiación ionizante que originan diversas fuentes de radiación naturales, y muy en particular conocer las principales fuentes de radiación gamma, ya que esta es la que tiene una menor energía pero un mayor alcance. Comparativamente, la radiación gamma tiene un alcance muy superior a las partículas beta o alfa, cuya capacidad de penetración es de unos micrometros y nanometros, respectivamente. A parte de estas fuentes de radiación debido a la existencia de radioisótopos en el suelo y el subsuelo, existe una exposición a radiación cósmica que depende de diversos factores como la latitud y la altitud, aunque su contribución global es menor (UNSCEAR, 2000).

En el medio natural, la principal fuente de radiación gamma son las rocas del substrato y los minerales del suelo (UNSCEAR, 2000). En el caso del interior de construcciones realizadas por el hombre, aparte de la radiación procedente del suelo y subsuelo, la principal fuente de radiación son los materiales de construcción, y particularmente lo materiales pétreos. Se incluyen en este grupo el uso de roca natural, materiales cerámicos y materiales aglomerantes como cementos, morteros y hormigones. La importancia de estos radica en que se estima que como promedio los seres humanos pasan alrededor de un $80 \%$ del tiempo de su vida en el interior de estas construcciones (vivienda y lugar de trabajo), al menos en países industrializados y climas templados, y por lo tanto expuestos a la radiación gamma emitida por los mencionados materiales (Moharram et al. 2011).

\subsection{Radioisótopos en las rocas (influencia de procesos geológicos)}

Los principales radioisótopos existentes en las rocas y los materiales del suelo son aquellos derivados de las series de desintegración del uranio $\left({ }^{238} \mathrm{U}\right.$ and $\left.{ }^{235} \mathrm{U}\right)$ y torio $\left({ }^{232} \mathrm{Th}\right)$, así como el potasio $\left({ }^{40} \mathrm{~K}\right)$. Aunque el U no está presente en los silicatos mas comunes (debido a su gran radio iónico) pueden producirse enriquecimientos de uranio en las fases finales de cristalización fraccionada de magmas, en pegmatitas o por fusion parcial de rocas sedimentarias o ígneas ácidas de la corteza, ya de por si comparativamente ricas en U. Las concentraciones medias estimadas de $\mathrm{U}$ son de aproximadamente $0,3 \mathrm{ppm}$ en basaltos, 3,8 ppm en granitos, 3,7 ppm en esquistos y 2,2 ppm en carbonatos, pudiendo llegar a 300 ppm en rocas fosfatadas. Bajo la forma de $\mathrm{U}^{+6}$ el uranio es muy reactivo y soluble en agua formando el ión uranilo $\left(\mathrm{UO}_{2}{ }^{+2}\right)$ presente en la mayor parte de especies minerales con U. En rocas cristalinas, el U es incorporado en minerales accesorios como xenotimo, circon, monacita, esfena y allanita. Al meteorizarse las rocas puede depositarse en el contorno de granos mineales y microfracturas como óxidos o silicatos de U (Dickson \& Scott, 1997).

El Th es 3-4 veces mas abundante que el $\mathrm{U}$ en la corteza terrestre al ser menos móvil y soluble en superficie excepto a pH ácido o pH neutro en presencia de compuestos orgánicos. Suele aparecer en monacita, torianita $\left(\mathrm{ThO}_{2}\right)$ y torita $\left(\mathrm{ThSiO}_{4}\right)$ entre otros (Gascoyne, 1992), o como elemento traza en fosfatos, óxidos y silicatos como en la allan- 
ita, xenótimo y circón, en concentraciones superiores a $1000 \mathrm{ppm}$, o en proporciones traza en otros minerales. Los procesos de meteorización pueden liberar Th por desagregación o puede quedar retenido en óxidos-hidróxidos de Fe y Ti o en arcillas.

El K aparece sobre todo en feldespatos (principalmente ortoclasa y microclina con un $13 \%$ de potasio) y micas (biotita y moscovita contienen alrededor de $8 \%$ en $\mathrm{K}$ ). Es relativamente abundante en rocas félsicas y escaso en las máficas (Dickson \& Scott, 1997). Durante la meteorización puede ser incorporado o absorbido en arcillas como la illita o la montmorillonita.

\subsection{Geoquímica de radionúclidos en Granitos}

Las rocas formadas en los últimos estadíos de implementación ígnea, como pegmatitas y aplitas retienen valores elevados de $\mathrm{K}$ y bajos de $\mathrm{U}$ y Th. Las rocas alcalinas están mas enriquecidas en Th que en U. Las rocas sedimentarias presentan una concentración en radionucleidos que refleja la roca que los origina por lo que su contenido en $\mathrm{U}$, Th y $\mathrm{K}$ es muy variable. Sin embargo, en las rocas metamórficas, se considera que el metamorfismo no afecta al contenido en radioelementos, aunque el contenido en $\mathrm{U}$ y Th tiende a disminuir con el grado de metamorfismo de facies apidoto-amfibólicas, para la facies granulítica. El rátio $\mathrm{Th} / \mathrm{U}$ disminuye al aumentar la temperatura y la presión (Heier, 1975).

En general, se considera que las rocas graníticas presentan valores elevados de $\mathrm{U}$ y Th, debido al magma que los origina, a movimientos tectónicos asociados a su génesis y a que las rocas generadas en la corteza suelen estar enriquecidas en radioelementos (Moura et al., 2011). La cantidad de U que puede exi- stir en un granitoide está determinada por sus minerales portadores, que en muchos granitos son accesorios (circón, esfena, xenótimo) pudiendo contener hasta un $85 \%$ del U.

Los procesos geológicos asociados a zonas de cizalla pueden provocar el enriquecimiento o empobrecimiento en radioelementos en rocas, dependiendo de si el cizallamiento es frágil o dúctil. El histórico de cristalización y los procesos de meteorización hidrotermal también pueden afectar a la distribución de los radionúclidos en rocas ígneas (Sroor et al, 2002). La formación de microbrechas en rocas sujetas a procesos de deformación ductil-frágil puede crear caminos a través de los cuales los fluidos enriquecidos en radionúclidos se mueven y subsecuentemente se depositan en rocas encajantes (Moura et al., 2011).

\subsection{Comportamiento de los radioisótopos en rocas}

En las series de desintegración de U y Th existen varios elementos que producen radiación gamma de forma significativa (con energía e intensidad suficiente) como para ser estimada su actividad por espectrometría gamma (IAEA, 2003). En rocas no alteradas, estas series se encontrarán en estado de equilibrio al ser sistemas cerrados. Si una serie con un isótopo padre de vida larga no se ve perturbada durante un tiempo aproximadamente 8 veces superior al período de vida media del isótopo hijo con mayor vida media, se considera que ambos miembros de la serie se desintegran a la misma tasa y por lo tanto están en equilibrio. La pérdida o movilización de algunos de estos elementos ocasiona un desequilibrio, que raramente ocurre en la serie del Th $\left({ }^{232} \mathrm{Th}\right)$ pero puede ser frecuente en la serie del ${ }^{238} \mathrm{U}$. (tabla 1). 


\begin{tabular}{|c|c|c|c|c|c|}
\hline \multicolumn{3}{|c|}{${ }^{238} \mathrm{U}$ serie } & \multicolumn{3}{|c|}{${ }^{232}$ Th Serie } \\
\hline Isotope & Vida media & Desintegración & Isotope & Vida media & Desintegración \\
\hline${ }^{238} \mathrm{U}$ & $4.468 \times 10^{9}$ años & alpha & ${ }^{232} \mathrm{Th}$ & $1.405 \times 10^{10}$ años & alpha \\
\hline${ }^{234} \mathrm{Th}$ & 24.1 días & beta & ${ }^{228} \mathrm{Ra}$ & 5.75 años & beta \\
\hline${ }^{234} \mathrm{~Pa}$ & $1.17 \mathrm{~min}$ & beta & ${ }^{228} \mathrm{Ac}$ & 6.25 horas & beta \\
\hline${ }^{234} \mathrm{U}$ & $2.48 \times 10^{5}$ años & alpha & ${ }^{228} \mathrm{Th}$ & 1.916 años & alpha \\
\hline${ }^{230} \mathrm{Th}$ & $7.7 \times 10^{4}$ años & alpha & ${ }^{224} \mathrm{Ra}$ & 3.6319 días & alpha \\
\hline${ }^{226} \mathrm{Ra}$ & 1600 años & alpha & ${ }^{220} \mathrm{Rn}$ & 55.6 días & alpha \\
\hline${ }^{222} \mathrm{Rn}$ & 3.82 días & alpha & ${ }^{216} \mathrm{Po}$ & $0.145 \mathrm{seg}$ & alpha \\
\hline${ }^{218} \mathrm{Po}$ & $3.05 \mathrm{~min}$ & alpha & ${ }^{212} \mathrm{~Pb}$ & 10.64 horas & beta \\
\hline${ }^{214} \mathrm{~Pb}$ & $26.8 \mathrm{~min}$ & beta & ${ }^{212} \mathrm{Bi}$ & $60.55 \mathrm{~min}$ & alpha+beta \\
\hline${ }^{214} \mathrm{Bi}$ & $19.8 \mathrm{~min}$ & beta & ${ }^{212} \mathrm{Po}$ & $299 \mathrm{nseg}$ & alpha \\
\hline${ }^{214} \mathrm{Po}$ & $162 \mu \mathrm{seg}$ & alpha & ${ }^{208} \mathrm{Tl}$ & $3.053 \mathrm{~min}$ & beta \\
\hline${ }^{210} \mathrm{~Pb}$ & 22.3 años & beta & ${ }^{208} \mathrm{~Pb}$ & estable & \\
\hline${ }^{210} \mathrm{Bi}$ & 5.01 días & beta & & & \\
\hline${ }^{210} \mathrm{Po}$ & 138.4 días & alpha & & & \\
\hline${ }^{206} \mathrm{~Pb}$ & estable & & & & \\
\hline
\end{tabular}

Tabla 1. Principales isótopos de la serie de desintegración del ${ }^{238} \mathrm{U} \mathrm{y}^{232} \mathrm{Th}$, vida media y tipos de desintegración.

Como ejemplo, en la serie del ${ }^{238} \mathrm{U}$, uno de sus isótopos hijos es fácilmente movilizado y lavado debido a su solubilidad ya que la desintegración de ${ }^{238} \mathrm{U}$ a ${ }^{234} \mathrm{Th}$ origina una ruptura en el cristal del que el ${ }^{238} \mathrm{U}$ formaba parte y quedando los isótopos hijos subsiguientes libres. Otros isótopos hijos como el ${ }^{226} \mathrm{Ra}$ también son fácilmente movilizados y lavados mientras que el ${ }^{222} \mathrm{Rn}$, originado por su desintegración, se pierde fácilmente por emanación al ser un gas, sobre todo dependiendo de la porosidad de la roca, que aumenta con la meteorización. De hecho, el ${ }^{226} \mathrm{Ra}$ es el isótopo considerado como radiológicamente mas importante, y se utiliza incluso como referencia en lugar del U (Kovler, 2007). En general se considera que las concentraciones medias de $\mathrm{Ra}$, Th y K en la corteza terrestre son de $40 \mathrm{~Bq} /$ $\mathrm{kg}, 40 \mathrm{~Bq} / \mathrm{kg}$ y $400 \mathrm{~Bq} / \mathrm{kg}$, respectivamente (EC, 1999).

\section{RADIACTIVIDAD EN MATERIALES DE CONSTRUCCIÓN}

El uso de materiales pétreos en la construcción puede derivar en una elevada exposición a radiación gamma e incluso $\mathrm{Rn}$ en el interior de construcciones, debido a la elevada radiactividad de los materiales. Para estimar la dosis gamma efectiva en el interior de una construcción se considera un coeficiente de conversión de dosis absorbida en el aire y un factor de ocupación del interior. La UNSCEAR (2000) considera que el coeficiente de conversión es de 0.7 Sv/Gy mientras que el factor de ocupación es de 0.8 (que corresponde al $80 \%$ del tiempo de vida que se pasa en la vivienda, considerado adecuado para países industrializados y de climas templados). La dosis efectiva anual media a nivel mundial es de $0.48 \mathrm{mSv}$ siendo el rango de 0,3-0,6 $\mathrm{mSv}$. 


\subsection{Consideraciones de tipo legal}

A pesar de estas consideraciones, no existe una regulación todavía bien desarrollada en los países de la Unión Europea acerca de la exposición a radiación gamma, a pesar del riesgo que supone para la salud (UNSCEAR, 2000), aunque en los últimos años se han dado pasos importantes para su desarrollo. La legislación mas antigua en Europa corresponde a restricciones fijadas por Finlandia para la exposición a radiación en edificios en 1991 (Markkanen, 1995). En 1999, la Unión Europea establece la European Basic Safety Standards Directive (BSS) restringiendo la exposición laboral a fuentes de radiación naturales pero sin líneas claras y dejando a los estados miembros el desarrollo de la legislación restringiendo la exposición a radiación. Esto ha llevado a algunos estados miembros a establecer restricciones en el uso de materiales de construcción. En general, se contempla para la UE que las dosis efectivas superiores a $1 \mathrm{mSv} / \mathrm{a}$ deben ser consideradas y aceptadas en casos excepcionales en materiales utilizados de forma local, recomendándose una dosis efectiva de 0,3-1 mSv/a (EC, 1999).

En el año 2013 el Consejo de la Unión Europea aprobó la Directiva de la UE (Directiva 2013/59/EURATOM del Consejo de 5 de diciembre de 2013) por la que se establecen las normas de seguridad básicas para la protección contra los peligros derivados de la exposición a radiaciones ionizantes (CUE, 2013) que entró en vigor en 2014. En ella se da un plazo de 4 años a los países miembros para establecer una legislación nacional al respecto. Una parte importante de la Directiva se refiere a los materiales de construcción (articulo 75, Anexos VIII y XIII). De acuerdo con esta norma existirá una obligación de medir la actividad específica de radioisótopos en estos materiales por los fabricantes utilizando el índice de actividad, debiendo estos cumplir una serie de requisitos.

Varios países miembros de la UE han establecido ya normativas de control a este respecto, como Finlandia, Polonia y la República Checa, utilizando como base el índice de actividad gamma (Rosen, 2014). Esta regulación se extiende para limitar los niveles de contenidos en radionucleidos en materiales de construcción que puedan ser utilizados en techos, muros y pavimentos de viviendas o espacios habitables en el caso de la República Checa. Los criterios adoptados son mas estrictos que los de la RP 112, estableciéndose que los materiales que sobrepasen ciertos límites solo podrán ser utilizados en casos justificados. También la legislación de Polonia (junto con la de la República Checa) limitan el contenido en ${ }^{226} \mathrm{Ra}$ en materiales de construcción, con la intención de limitar la exposición a Rn (Rosen, 2014).

\subsection{Consideraciones metodológicas}

Para evaluar el riesgo de exposición a radiación gamma debido al uso de materiales de construcción con una concentración de isótopos radiactivos determinada se utilizan los índices de actividad, que normalmente se basan en la medición de las concentraciones de ${ }^{226} \mathrm{Ra},{ }^{232} \mathrm{Th}$ y ${ }^{40} \mathrm{~K}$, aunque en casos especiales pueden considerarse otros núclidos como el ${ }^{137} \mathrm{Cs}$, como por ejemplo en el uso de cenizas como aditivo en morteros y hormigones (Markkanen, 1995). Los índices de actividad deben considerar también la forma del espacio interior y la cantidad de cada material usado en la construcción. Un ejemplo es el índice gamma $\left(I_{\gamma}\right)$ indicado por 
la Comisión Europea, aunque existen otros como el índice alfa, estimado a partir del ${ }^{226} \mathrm{Ra}$ (EC, 1999; Righiy y Bruzzi, 2006).

En términos genéricos se consideran dos métodos para evaluar la exposición externa a radionúclidos naturales (UNSCEAR, 2000). Uno (indirecto) consiste básicamente en resumir directamente las tasas de dosis gamma externas medidas en el aire, en interior y exterior, substrayendo la tasa de dosis debida a la radiación cósmica. El otro (directo) consiste en calcular la tasa de dosis gamma externa en el aire por medio de mediciones de las concentraciones de radionúclidos en el suelo.

En los últimos años se han realizado medidas directas de las tasas de dosis absorbida en el aire por todo el mundo, abarcando aproximadamente al $70 \%$ de la población mundial (UNSCEAR, 2000), observándose rangos de 10-200 nGy/h con medias que varían entre 18 y $93 \mathrm{nGy} / \mathrm{h}$, dependiendo de la litología y los materiales de construcción utilizados. En las medidas indirectas se han considerado diferentes coeficientes de transformación de la actividad de los isótopos en los materiales a la tasa de dosis absorbida (UNSCEAR, 2000).

\section{4. ÁREA Y OBJETO DE ESTUDIO}

En el NW de Portugal existe un importante volumen de granitoides correspondientes (Dias et al., 2002) a sucesivos pulsos magmáticos, principalmente correspondientes a la etapa postcolisional de la orogenia varisca (290-321 Ma). La composición y tipología de las rocas granitoides es muy variable agrupándose estos en cuatro tipos: granitoides sin-D3 (monzogranitos/granidoiritas biotíticos y de dos micas), granitoides tardi-D3 (monzogranitos/granidoiri- tas esencialmente biotíticos), granitoides tardi a post-D3 (leucogranitos de dos micas muy peraluminosos) y granitoides post-D3 (granitos biotíticos a biotítico-moscovíticos).

La ciudad de Braga se localiza en la zona litoral norte, asentada en el conocido como granito de Braga que forma parte del complejo granítico de Braga. El granito de Braga ha sido usado con mucha frecuencia en la construcción de edificios a lo largo de la historia de la Ciudad para pavimentos, muros y techos. Por ello, existe una gran cantidad de edificios construidos, al menos parcialmente, con este granito. Es un granito rico en biotita, de grano fino a medio y ligeramente porfírico (Dias et al., 2002). Esta compuesto aproximadamente por un $22-27 \%$ de cuarzo, 27-32\% de plagioclasa, 22-31 de feldespato potásico, $10-19 \%$ de biotita y $0-4 \%$ de biotita (Dias et al., 2002). Un estudio de campo de la roca permite observar su afloramiento en cantera en al menos 5 estados diferentes de meteorización. De estos, se ha considerado que el estado del 1 al 3, los mas utilizados como material de construcción, son los menos alterados.

Dentro de un programa nacional de medida de las dosis de radiación gamma natural en Portugal, Amaral et al. (1992) estimaron que la media aritmética de la tasa de dosis absorbida para el distrito de Braga es de 152,2 $\mathrm{nGy} / \mathrm{h}$ con un intervalo de $95,5-226,5 \mathrm{nG} / \mathrm{h}$, siendo la dosis efectiva anual exterior e interior de $0,19 \mathrm{mSv} / \mathrm{a}$ y 0,9 mSv/a, respectivamente. En el Mapa Radiológico para el ayuntamiento de Braga, la tasa de dosis medida es superior a $90 \mathrm{nGy} / \mathrm{h}$ dentro de un intervalo de 54-211,7 nGy/h (Amaral, 2000; AIEA, 2003), dándose los valores mas elevados en suelos originados a partir de rocas intrusivas y menor en los de rocas metamórficas (esquistos). 
El objetivo de este estudio es evaluar la radiactividad gamma del granito de Braga como material de construcción. Dado que su uso durante la historia de la ciudad ha sido extenso, en la actualidad existe una gran cantidad de estructuras históricas en las que ha sido utilizado este tipo de roca, lo que implica la necesidad de evaluar la exposición del público a la radiactividad gamma, tanto en viviendas como en zonas de trabajo situados en el área monumental de la ciudad. Para ello, se ha estimado la actividad de varios radioisótopos en muestras de granito de 5 grados de meteorización y se han utilizado los datos para calcular el índice gamma para materiales de construcción (gamma building index o $I_{\gamma}$ ) recomendado por la Guia Técnica de la UE (RP112) para el control legal de materiales de construcción (EC, 1999). Además, se han calculado otros dos parámetros utilizados para estimar la peligrosidad radiológica de los materiales como son la Actividad equivalente promedio de Radio $\left(\mathrm{Ra}_{\mathrm{eq}}\right)$ y la dosis equivalente anual gonadal (AGED).

\section{MATERIAL Y MÉTODOS}

El estudio realizado en este trabajo comprende dos partes: un análisis estimativo de la meteorización de diferentes muestras del granito de Braga y una análisis de actividad de los isótopos de $\mathrm{U}$, Th y $\mathrm{K}$ o radioisótopos de sus correspondientes series de desintegración $(\mathrm{Ra})$.

\subsection{Estimación del grado de meteorización}

Como es conocido, la meteorización disminuye la resistencia mecánica y cohesión de las rocas. Esta característica fue utilizada para estimar la meteorización de diferentes muestras del granito de Braga, tras una primera estimación de la meteorización utilizando sus variaciones cromáticas, observables desde un punto de vista macroscópico, dado que se observa un cambio de color de la roca hacia el amarillo en los grados 1 y 2 .

Para un análisis mas objetivo del grado de meteorización, se realizaron medidas de porosidad por medio del coeficiente de absorción de agua. Después de preparar probetas de roca correspondientes a los 5 estados de meteorización observados en afloramientos y en una cantera, estas se secaron en estufa. Una vez secas se sumergieron en agua desionizada y se midió su masa tras 72 horas, encontrándose las muestras saturadas de agua. Para determinar el coeficiente de absorción de agua a presión atmosférica se utilizó la ecuación (1):

$$
A_{b}=\left(m_{5}-m_{d}\right) \times 100 / m_{d}(1)
$$

siendo $m_{s}$ la masa saturada y $m_{d}$ la masa de la muestra seca.

\subsection{Espectrometría gamma}

Para el análisis de la actividad de isótopos de las series de U, Th y K se secaron en estufa las muestras y se molieron hasta obtener un tamaño de grano menor de 63 micras. Una vez molidas se calcinaron a $450^{\circ} \mathrm{C}$ para eliminar cualquier presencia de materia orgánica que pudiese haber en la superficie de las muestras o haber penetrado su interior. Las muestras fueron selladas durante 25 días hasta alcanzar el equilibrado del Rn y luego se realizaron los análisis de las muestras mediante un detector de semiconductor de Germanio tipo coaxial marca CANBERRA modelo GR6022. Este detector de radiación gamma se encuentra dentro 
de un blindaje de plomo de baja actividad de $10 \mathrm{~cm}$ de grosor, y conectado a un sistema de reducción de fondo en anticoincidencia basado en un detector de centelleador plástico. Sus características principales son una eficiencia relativa del $60 \%$, y una resolución de $1.05 \mathrm{keV}$ y $2.2 \mathrm{keV}$ para una energía de $122 \mathrm{keV}$ y $1332 \mathrm{keV}$, respectivamente.

La medida de emisores gamma de las muestras, introducidas en sus respectivas cajas petri de polipropileno y selladas adecuadamente, es una técnica no destructiva que permite analizar la concentración de actividad de los siguientes radioisótopos naturales: $\mathrm{Be}-7, \mathrm{~K}-40, \mathrm{Tl}-208, \mathrm{~Pb}-210, \mathrm{~Pb}-212$, Bi-212, Pb-214, Bi-214, Ra-226, Ac-228 y Th-234; y artificiales: Cr-51, Mn-54, Co-58, Co-60, Fe-59, Zn-65, Nb-95, Zr-95, Ru-103, Ru-106, I-131, Cs-134, Cs-137, Ba-140, La140, Ce-144, y Am-241. La actividad final de la muestra se calcula tras la calibración en eficiencia, corregida por autoabsorción y suma en coincidencia, de la geometría de recuento mediante simulación por Monte Carlo con los códigos GEANT4 y LABSOCS.

\section{3. Índices de concentración de actividad}

En términos generales, los índices son utilizados como una herramienta para evaluar la exposición a radiación gamma derivada de materiales de construcción. Consisten en la suma de las contribuciones de los radionúclidos que emiten radiación gamma presentes en los materiales evaluados. Las líneas maestras de la Comisión Europea (RP-112) constituyen el primer documento publicado (EC, 1999) que establece una serie de principios de protección radiológica respecto a la radiación gamma natural (externa e interna) en materiales de construcción (Moharram et al., 2011). Esta guía de protección radiológica establece los valores para materiales de construcción de 300,200 y $3000 \mathrm{~Bq} / \mathrm{kg}\left(\operatorname{Ref}_{i}\right)$ para las actividades de ${ }^{226} \mathrm{Ra}$ (serie del ${ }^{238} \mathrm{U}$ ), ${ }^{232} \mathrm{Th}$ y ${ }^{40} \mathrm{~K}$, respectivamente para el índice de concentración de actividad gamma $\left(I_{\gamma}\right)$. La Comisión Europea sugiere que los materiales de construcción deben estar exentos de toda restricción respecto a su radiactividad si el exceso de radiación gamma originado por ellos incrementa la dosis anual efectiva que una persona en $0,3 \mathrm{mSv}$ como máximo. El índice de concentración de actividad gama que resulta de la expresión (2):

$$
\mathrm{I}_{\gamma}=\sum\left(\mathrm{C}_{i} / \operatorname{Ref} f_{i}\right)
$$

Siendo $C_{i}$ la concentración del isótopo $i$ en $\mathrm{Bq} / \mathrm{kg}$ en la muestra de estúdio Ref $_{i^{*}}$

Otro índice usado con frecuencia es el índice de actividad de radio equivalente $\left(\mathrm{Ra}^{e q}\right)$. Este fue propuesto por Beretka y Mathew (1985) para limitar la radiactividad en materiales de construcción en base a la actividad del Ra, y ha sido utilizada extensamente, El $R a_{e q}$ se define como la suma ponderada de las concentraciones de actividad de ${ }^{226} \mathrm{Ra}$, ${ }^{232} \mathrm{Th}$ y ${ }^{40} \mathrm{~K}(3)$ :

$\mathrm{Ra}_{\mathrm{eq}}=\mathrm{C}_{\mathrm{Ra}}+1,43 \mathrm{C}_{\mathrm{Th}}+0,077 \mathrm{C}_{\mathrm{K}} \leq 370 \mathrm{~Bq} / \mathrm{kg}$

Los factores de ponderación para ${ }^{226} \mathrm{Ra}$, ${ }^{232} \mathrm{Th}$ y ${ }^{40} \mathrm{~K}$ son $1,1,43$ y 0,077 , respectivamente. El máximo valor de $R a_{e q}$ en materiales de construcción no debe sobrepasar $\operatorname{los} 370 \mathrm{~Bq} / \mathrm{kg}$ para un uso seguro, manteniéndose así la dosis externa por debajo de 1,5mSv/a (Serena, 2006).

El índice conocido como $A G E D$ (annual gonadal equivalent dose) se basa en el potencial peligro que supone la radiación gamma recibida en ciertos órganos. Las 
gónadas, la médula ósea activa y las células de la superficie de los huesos son considerados órganos de interés para el UNSCEAR (1988). La dosis equivalente anual gonadal para los residentes en una casa o trabajadores en un determinado edificio o ambiente en el cual existen materiales de construcción con una cierta concentración de radionúclidos se calcula a través de la expresión (4):

$A G E D(\mu \mathrm{Sv} / \mathrm{a})=3,09 \mathrm{C}_{\mathrm{Ra}}+4,18 \mathrm{C}_{\mathrm{Th}}+0,314 \mathrm{C}_{\mathrm{K}}$

siendo 3,09, 4,18 y 0,314 los factores de conversión para ${ }^{226} \mathrm{Ra},{ }^{232} \mathrm{Th} \mathrm{y}{ }^{40} \mathrm{~K}$, respectivamente. El modelo considerado en este parámetro se basa en una casa típica con paredes de espesor infinito, lo que permite comparar el $A G E D$ con una casa que contiene concentraciones de ${ }^{226} \mathrm{Ra},{ }^{232} \mathrm{Th}$ y ${ }^{40} \mathrm{~K}$ similares a las del promedio mundial en suelos (Arafa, 2004).

\section{RESULTADOS Y DISCUSIÓN}

La absorción de agua en las muestras de granito mostró una muy baja porosidad en las muestras con menor grado de alteración (1 y 2), algo mayor e la muestra afectada por una meteorización intermedia (3) y creciente en las muestras mas alteradas (4-5). En la tabla 2 se pueden observar los resultados. Por tanto, las observaciones macroscópicas son coherentes con estos tests.

\begin{tabular}{|c|c|c|c|c|c|}
\hline Muestra & $\mathbf{1}$ & $\mathbf{2}$ & $\mathbf{3}$ & $\mathbf{4}$ & $\mathbf{5}$ \\
\hline $\mathrm{A}_{\mathrm{b}}(\%)$ & 0.7 & 0.8 & 1.6 & 3.1 & 8.6 \\
\hline
\end{tabular}

Tabla 2. Resultados de los ensayos de absorción de agua del granito de Braga.

Los resultados de la espectrometría gamma se detallan en la tabla 3 . En esta se pueden observar las actividades específicas de varios isótopos de las series de desintegración de $\mathrm{U}$ y Th, así como la actividad específica del ${ }^{40} \mathrm{~K}$. Se observa que el menor valor medido para la actividad del ${ }^{238} \mathrm{U}$ es de $70 \pm 15 \mathrm{~Bq} / \mathrm{kg}$ en la muestra 4. El menor valor medido para la actividad del ${ }^{232} \mathrm{Th}$ es de $34 \pm 2 \mathrm{~Bq} / \mathrm{kg}$ en la muestra 2 mientras que la actividad mas baja para el ${ }^{40} \mathrm{~K}$ se observa en la muestra 1. En términos generales, se observa que la concentración de radionúc- lidos en las muestras del granito de Braga están por encima de los promedios mundiales para materiales de construcción, que son de 50,50 y $500 \mathrm{~Bq} / \mathrm{kq}$ para ${ }^{238} \mathrm{U},{ }^{232} \mathrm{Th}$ $\mathrm{y}{ }^{40} \mathrm{~K}$, respectivamente (UNSCEAR, 2000). La única excepción a esto se observa en la muestra 2 para el ${ }^{232} \mathrm{Th}$. También se observa una tendencia muy leve al descenso de la actividad del ${ }^{238} \mathrm{U}$ y un claro incremento de la concentración de ${ }^{232} \mathrm{Th}$ con la meteorización. Igualmente, parece observarse un incremento en la concentración de ${ }^{40} \mathrm{~K}$ con la meteorización. 


\begin{tabular}{|l|l|l|l|}
\hline Muestra & $\begin{array}{l}\text { Actividad específica }{ }^{238} \mathbf{U} \\
(\mathbf{B q} \mathbf{~ K g}-\mathbf{1})\end{array}$ & $\begin{array}{l}\text { Actividad específica }{ }^{232} \mathbf{T h} \\
\left(\mathbf{B q ~ K g}^{-1}\right)\end{array}$ & $\begin{array}{l}\text { Actividad específica } \\
\left(\mathbf{B q ~ K g}^{-1} \mathbf{~} \mathbf{K}\right.\end{array}$ \\
\hline 1 & $139 \pm 29$ & $95 \pm 4$ & $1305 \pm 60$ \\
\hline 2 & $71 \pm 16$ & $34 \pm 2$ & $2080 \pm 87$ \\
\hline 3 & $114 \pm 25$ & $70 \pm 5$ & $1681 \pm 72$ \\
\hline 4 & $70 \pm 15$ & $202 \pm 10$ & $1570 \pm 68$ \\
\hline 5 & $110 \pm 22$ & $202 \pm 8$ & $1567 \pm 65$ \\
\hline
\end{tabular}

Tabla 3. Actividades específicas de ${ }^{238} \mathrm{U},{ }^{232} \mathrm{Th}$ y ${ }^{40} \mathrm{~K}$ ara las muestras.

ATENÇÃO que no artigo para Sevilha havia um erro no valor de $40-\mathrm{K}$ para a primeira amostra (e que depois foi corrigido na dissertação) OK

El cálculo de los diferentes índices utilizados para evaluar el riesgo derivado del uso del granito de Braga como material de construcción revela la existencia de una correlación entre el grado de meteorización de los materiales y los índices obtenidos. Los valores de índice gamma $\left(I_{\gamma}\right)$ obtenidos para las muestras con diferente grado de meteorización se pueden observar en la tabla 3 . Estos resultados indican que las cinco muestras exceden el valor umbral de 1 , recomendado para el uso como materiales de construcción por lo que debería considerarse un estudio mas profundo de éstos en relación a este índice dado su extenso uso en edificios históricos de la ciudad de Braga. El valor del índice se incrementa además con el grado de meteorización, siendo el mínimo calculado de 1,1 y el máximo de 1,9.

Los índices de actividad de radio equivalente $\left(R a_{e q}\right)$ obtenidos están ligeramente por debajo o en el entorno del límite propuesto de $370 \mathrm{~Bq} / \mathrm{kg}$ para un uso seguro en las muestras menos meteorizadas (el valor más elevado es $375 \mathrm{~Bq} / \mathrm{kg}$ ). Sin embargo, en las muestras que presentan un mayor grado de meteorización (4-5) se observa un incremento en este índice, de modo que sobrepasa ese valor de forma significativa. De un modo similar, el cálculo de la dosis equivalente anual gonadal muestra una variación importante acorde al grado de meteorización. Además, todas las muestras muestran valores elevados de $A G E D$ en comparación con la media mundial.

\begin{tabular}{|c|c|c|c|}
\hline Muestra & Índice gamma $\left(I_{\square}\right)$ & Radio Equivalente $\left(R a_{e q}\right)\left(\mathbf{B q} \mathbf{~ k g}^{-1}\right)$ & $A G E D$ \\
\hline 1 & 1.37 & 375 & 1236 \\
\hline 2 & 1.10 & 280 & 1015 \\
\hline 3 & 1.29 & 344 & 1173 \\
\hline 4 & 1.77 & 480 & 1554 \\
\hline 5 & 1.90 & 520 & 1676 \\
\hline
\end{tabular}

Tabla 4. Parametros relacionados con la radiación gamma calculada a partir de las actividades de $222 \mathrm{Ra}, 232 \mathrm{Th}$ y $40 \mathrm{~K}$ en las muestras estudiadas. HÁ UMAS PEQUENAS CORREÇÕES NA PRIMEIRA AMOSTRA 


\section{CONCLUSIONES}

El estudio de los radionúclidos emisores de radiación gamma en muestras de granito de Braga indican que esta roca tiene en términos genéricos una actividad específica de ${ }^{226} \mathrm{Ra},{ }^{232} \mathrm{Th}$ y ${ }^{40} \mathrm{~K}$ por encima de los valores promedios a nivel mundial para los materiales de construcción. En este estudio se observa que además existe un incremento en los valores de los índices relativos a la radiación gamma en estos materiales cuando están netamente meteorizados. El estudio de 5 muestras con diferente grado de meteorización muestra que todas ellas exceden el valor de $I_{\gamma}$ indicado como límite por la CE (CUE, 2013), o sea, estos valores implicarían estudios detallados de los edificios donde este granito sea usado. Sin embargo, las recomendaciones de EC 112 indican que estos granitos no suponer un problema cuando utilizados como revestimientos superficiales (CE, 1999). El uso de otros indicadores como la dosis equivalente anual gonadal ( $A G E D)$ muestra un resultado muy similar al del índice anterior. Sin embargo, respecto a otros indicadores, se observa que las muestras no meteorizadas o poco meteorizadas no exceden (o exceden muy poco) el índice $R a_{\text {eq }}$ promedio y recomendado para materiales de construcción.

A partir de estos resultados, y dado el extenso uso que ha tenido este tipo de roca en edificios y estructuras históricas de la ciudad de Braga, con el consiguiente riesgo de exposición del publico a estos materiales, parece recomendable la realización de un estudio de los niveles de radiación gamma actuales in situ, en diversos edificios de la ciudad. Este estudio permitiría determinar si existen casos de una excesiva exposición de personas a radiación gamma con el con- siguiente riesgo, ya que existen una serie de parámetros que solo un estudio de este tipo pueden considerar (Risica et al., 2001).

\section{REFERENCIAS}

AIEA (2003) Internatinal Atomic Energy Agency: Guidelines for radioelement mapping using gamma ray spectrometry data. IAEA-TECDOC-1363, Vienna.

Amaral, E. Alves J., Carreiro J. 1992. Doses to the Portuguese population die to natural gamma radiation. Radiation protection dosimetry: 541-543.

Amaral, E.M. (2000) Natural gamma radiation in air versus soil nature in Portugal. Procedings IRPA, 10, Hirosima, P-1, P-12.

Arafa, W. 2004. Specific activity and hazards of granite samples collected from the Eastern Desert of Egypt. Journal of Environmental Radioactivity 75, 315-327.

Beretka, J., Mathew, P.J. (1985) Natural radioactivity of Australian building materials, industrial wastes and by-products. Health Pysics, 48, 87-95.

CUE - Consejo de la Union Europea (2013) - Directiva 2013/59/EURATOM del Consejo de 5 de diciembre de 2013 por la que se establecen normas de seguridad básicas para la protección contra los peligros derivados de la exposición a radiaciones ionizantes, y se derogan las Directivas 89/618/Euratom, 90/641/Euratom, 96/29/Euratom, 97/43/Euratom y 2003/122/Euratom

Dias, G., Sirngesl, P. P., Ferreira, N., \& Leterrier, J. (2002). Mantle and Crustal Sources in the Genesis of LateHercynianGranitoids (NW Portugal ): Geochemical and Sr-Nd Isotopic Constraints. Gondwana Research, 287-305. 
Dickson, B.L., Scott, K.M. (1997) Interpretation of aerial gamma-rays surveys - adding the geochemical factors. Australia. AGSO, Journal of Australian Geology and Geophysics, 17(2), 187200.

E.C. - European Comission. 1999. Radiation protection 112 Radiological Protection Principles concerning the Natural Radioactivity of Building Materials. Luxembourg.

Gascoyne, M. (1992) Geochemistry of the actinides and their daughters. In: Ivanovich, M, Harmon, R.S., (eds.). Uranium series disequilibrium, aplications to Earth, marine and environmental sciences. Clarendon Press, Oxford, 34-61.

Heier, K.S. (1975) The movement of uranium Turing higher grade metamorphic processes. Theoretical and practical aspects of uranium geology. Philosophical Transactions of the Royan Society of London, Series A, Mathematical and Physical Sciences, 291(1381), 413421.

Kovler, K. 2009. Radiological constraints of using building materials and industrial by-products in construction. Construction and Building Materials, 23(1), 246253. Haifa, Israel.

Markkanen, M. (1995). Radiation Dose Assessments for Materials with elevated Natural Radioactivity. STUK-BSTO 32, Finnish Center for Radiation and $\mathrm{Nu}-$ clear Safety. Helsinki.

Moharram B., Suliman M., Zarhan N., Shennawy S., El Sayed A. External exposure doses due to gamma emitting natural radionuclides in some Egyptian building materials. Applied Radiation and Isotopes 2011; 70 (2012): 241-248
Moura, C.L., Artur, A.C., Bonotto, D.M., Guedes, S., Martinelli, C.D. (2011) Natural radioactivity and radon exhalation rate in Brazilian igneous Rocks. Applied Radiation and Isotopes: including data, instrumentation and methods for use in agriculture, industry and medicine, 69(7), 1094-1099.

Righi, S., Bruzzi, L. (2006) Natural radioactivity and radon exhalation in building materials used in Itallian dwellings. Jorunal of Environmental Radioactivity, 88, 158-170.

Risica, S., Bolzan, C., Nuccetelli, C. 2001. Radioactivity in building materials: room model analysis and experimental methods. The Science of the total environment, 272(1-3), 119-26.

Rosen, D. (2014) Radiation from mansonry products - Dose assessment and classifications of emited gamma radiation. $9^{\text {th }}$ International mansonry conference, Guimaraes, Portugal.

Sroor, A., Afifi, S.Y., Abdel-Haleem, A.S., Salman, A.B., Abdel-Sammad, M. (2002) Environmental pollutant isotope measurements and natural radioactivity assessment ofr North Tushki area, Southwestern Egypt. Applied Radiation Isotopes, 57 (3), 427-436.

Sonkawadea, R.G., Kantb, K., Muralithara, S., Kumara, R., Ramola, R.C., 2008. Natural radioactivity in common building construction and radiation shielding materials.Atmospheric Environment 42, 2254-2259

UNSCEAR - United Nations Scientific Committee on the Effects of Atomic Radiation. 1988. New York.

UNSCEAR - United Nations Scientific Committee on the Effects of Atomic Radiation. 2000. New York. 